\title{
Restoring the equivalence between the light-front and manifestly covariant formalisms
}

\author{
Bernard L. G. Bakker, ${ }^{1}$ Martin A. DeWitt, ${ }^{2}$ Chueng-Ryong Ji, ${ }^{2}$ and Yuriy Mishchenko ${ }^{3}$ \\ ${ }^{1}$ Department of Physics and Astronomy, Vrije Universiteit, Amsterdam, The Netherlands \\ ${ }^{2}$ Department of Physics, Box 8202, North Carolina State University, Raleigh, North Carolina 27695-8202, USA \\ ${ }^{3}$ Cold Spring Harbor Laboratory, 1 Bungtown Road, Cold Spring Harbor, New York 11724, USA
}

(Received 7 September 2005; published 21 October 2005)

\begin{abstract}
We discuss a treacherous point in light-front dynamics (LFD) which should be taken into account to restore complete equivalence with the manifestly covariant formalism. We present examples that require an inclusion of the arc contribution in the light-front energy contour integration in order to achieve the equivalence between the LFD result and the manifestly covariant result.
\end{abstract}

PACS numbers: 11.10.-z, 11.30.Cp, 11.40.- $\mathrm{q}$

\section{INTRODUCTION}

It is well known that spurious divergences appear in light-front calculations that do not appear when the same amplitudes are computed in the standard equal-time formulation. This has been taken to mean that the equivalence between the light-front and manifestly covariant formalisms is not complete. These divergences have been regulated with various methods including the principal-value prescription, the Mandelstam-Leibbrandt prescription, cutoffs, smearing, and BPHZ-like differentiation and reintegration. We encountered an example of such a divergence in one of our previous works [1]. There, an end-point singularity was seen in the $(1+1)$-dimensional calculation of the pseudoscalar (and scalar) elastic form factor. The form factor was computed by first writing down the oneloop, triangle Feynman diagram and then integrating over the light-front energy by the method of residues. When the plus component of the current was used, the form factor was finite and equal to the manifestly covariant result. However, when the minus component of the current was used, an end-point singularity appeared in the result. Once this singular piece was removed by hand, the remaining amplitude was equal to the manifestly covariant result. In a subsequent paper [2] it was shown that this end-point singularity could be removed by smearing the photon vertex, which introduces a cutoff parameter, $\Lambda$. The singularity was removed for finite values of $\Lambda$, as well as in the limit $\Lambda \rightarrow \infty$.

However, further investigation has shown that when such light-front calculations are handled properly, spurious divergences do not appear in the final result and there is no need for any regularization procedure. Thus far, such divergences have appeared because of the application of the naive method of performing the loop integrations in which one simply picks up the residues, and ignores the contribution along the arc used to close the contour at infinity. In the ordinary equal-time (or instant form) Hamiltonian formalism, this procedure is safe unless one utilizes a common trick to reduce the number of denominators because the structure of the propagators is such that the integrand will always vanish sufficiently fast as the energy $\left(k^{0}\right)$ goes to infinity. Consequently, the contribution along the arc at infinity vanishes and the full result is simply given by the sum of the residues. However, in the light front there are cases in which the integrand does not vanish sufficiently fast as the light-front energy $\left(k^{-}\right)$goes to infinity. Then, the arc contributions are nonzero and must be included in order to obtain the correct result. Moreover, we show in this work that the point contributions occur when the integration contour in $k^{-}$plane crosses a moving singularity.

In a paper devoted to the discussion of the equivalence of light-front (LF) and covariant QED, Misra and Warawdekar [3] also spotted the "pole at infinity" where we use arc and point contributions. Their treatment, based on the method of Ref. [4], completely restores equivalence at the one-loop level. We also notice similar considerations of finding counterterms in LF Hamiltonians. In the classic paper [5] where the discretized light cone quantization (DLCQ) was introduced, the self-induced inertias were found when the normal ordered Hamiltonian was considered. Later, Burkardt [6] constrained the finite part of noncovariant counterterms in effective LF Hamiltonians.

In this work, in order not to obscure the main issue, we will investigate the $(1+1)$ dimensional calculations of both a simple vector two-point function (Sec. II) and the pseudoscalar charge form factor (Sec. III). Extensions to the $(3+1)$ dimensional calculations are straightforward. In Sec. II A we will show how neglecting the arc contributions at infinity leads to the appearance of an end-point singularity in the minus component of the vector two-point function. We will also show that computing these arc contributions is not an easy task. Computation involves a delicate limit and should be treated carefully. Therefore, in Secs. II B and II C, we will introduce different methods of evaluating the integral which include the arc contributions, but do not require that they be computed explicitly. Each of these procedures involves reducing $n$-point functions to combinations of $m$-point functions with $m<n$ [7], and each removes the end-point singularity and restores the equivalence between the manifestly covariant and lightfront results. In Sec. IID we will show the similarities 
between these methods and the tensor method which involves reducing a general integral to a sum of scalar $n$-point functions. In Sec. III, we will show how each of the methods of Secs. II B and IIC can be applied to the calculation of the pseudoscalar charge form factor, restoring the equivalence between the form factor obtained from the minus component of the current and that obtained in a manifestly covariant way. Finally, we will relate our findings to the previous counterterm considerations $[5,6]$ and finish with some concluding remarks in Sec. IV. In the appendix, we present details of the calculation used in Sec. II B.

\section{THE VECTOR TWO-POINT FUNCTION}

\section{A. Origin of the end-point singularity}

To show how the problem of the end-point singularity arises and to discuss how it should be resolved, we will use the simple example of the vector two-point function in $(1+$ 1) dimensions,

$$
V_{k_{1}(k-p)_{2}}^{\mu}=\int d^{2} k \frac{k^{\mu}}{\left[k^{2}-m_{1}^{2}+i \epsilon\right]\left[(k-p)^{2}-m_{2}^{2}+i \epsilon\right]} .
$$

This two-point function could arise, for example, in the calculation of the fermion self-energy (see Fig. 1). Upon integration we find the form $V_{k(k-p)}^{\mu}=I p^{\mu}$. In terms of a Feynman parameter, $x$, the manifestly covariant result for $I$ is given by

$$
I_{\mathrm{cov}}=-i \pi \int_{0}^{1} d x \frac{x}{D_{\mathrm{cov}}},
$$

where the covariant denominator function is

$$
D_{\text {cov }}=x(1-x) p^{2}-(1-x) m_{1}^{2}-x m_{2}^{2} .
$$

To evaluate the same integral in the light front, it is necessary to rewrite it in terms of the light-front energy $\left(k^{-}=k^{0}-k^{1}\right)$ and momentum $\left(k^{+}=k^{0}+k^{1}\right)$, and then perform the integration in the complex $k^{-}$plane. Here, $k^{2}=k^{+} k^{-}$and so

$$
V_{k_{1}(k-p)_{2}}^{\mu}=\frac{1}{2} \int d k^{+} d k^{-} \frac{k^{\mu}}{D_{1} D_{2}},
$$

where we have used the denominators

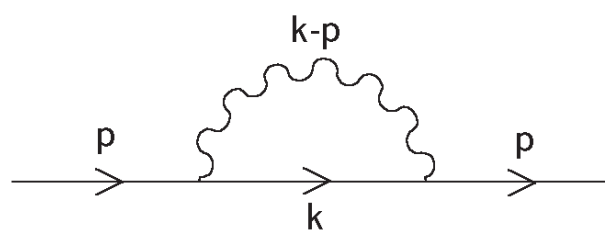

FIG. 1. The self-energy diagram.

$$
\begin{aligned}
D_{1} & =k^{2}-m_{1}^{2}+i \epsilon=k^{+} k^{-}-m_{1}^{2}+i \epsilon=k^{+}\left(k^{-}-k_{1}^{-}\right), \\
D_{2} & =(k-p)^{2}-m_{2}^{2}+i \epsilon \\
& =\left(k^{+}-p^{+}\right)\left(k^{-}-p^{-}\right)-m_{2}^{2}+i \epsilon \\
& =\left(k^{+}-p^{+}\right)\left(k^{-}-k_{2}^{-}\right),
\end{aligned}
$$

and where $k_{1}^{-}=\left(m_{1}^{2}-i \epsilon\right) / k^{+}$and $k_{2}^{-}=p^{-}+\left(m_{2}^{2}-\right.$ $i \epsilon) /\left(k^{+}-p^{+}\right)$. Let us first examine the plus component $(\mu=+)$. Since the positions of the two poles depend on the value of $k^{+}$we must first break the range of the $k^{+}$ integration into different regions. For $k^{+}<0$, both $k_{1}^{-}$and $k_{2}^{-}$are located in the upper half-plane. For $0 \leq k^{+} \leq p^{+}$, $k_{1}^{-}$shifts to the lower half-plane. And finally for $k^{+}>p^{+}$, both poles are located in the lower half-plane. For the first region $\left(k^{+}<0\right)$ and the third region $\left(k^{+}>p^{+}\right)$we can close the contour in the lower and upper half-planes, respectively, so the result of these integrations is zero. This leaves only the second region $\left(0 \leq k^{+} \leq p^{+}\right)$where, if we pick up the residue of the pole $k_{1}^{-}$we find the lightfront result to be,

$$
I_{\mathrm{LF}^{+}}=-i \pi \int_{0}^{1} d x \frac{x}{D_{\mathrm{cov}}} .
$$

Here, we have used the definition $k^{+}=x p^{+}$. This is clearly identical to the manifestly covariant result of Eq. (2).

Now, for the minus component $(\mu=-)$, the integration is a bit more tricky. If we naively follow the same procedure as for the plus component, we would say that the integration in the first and third regions is zero. In the second region, if we pick up the residue of the $k_{1}^{-}$pole we would obtain (again with $k^{+}=x p^{+}$)

$$
\begin{aligned}
I_{\mathrm{LF}^{-}}^{\text {residue }} & =-\frac{i \pi}{p^{2}} \int_{0}^{1} d x \frac{m_{1}^{2}}{x\left[x(1-x) p^{2}+x\left(m_{1}^{2}-m_{2}^{2}\right)-m_{1}^{2}\right]} \\
& =-\frac{i \pi}{p^{2}} \int_{0}^{1} d x \frac{(1-x) p^{2}+m_{1}^{2}-m_{2}^{2}}{D_{\mathrm{cov}}}+\frac{i \pi}{p^{2}} \int_{0}^{1} \frac{d x}{x},
\end{aligned}
$$

which is clearly not the same as $I_{\mathrm{cov}}$ and $I_{\mathrm{LF}^{+}}$. In fact, this result involves an end-point singularity at $x=0$. In the second line above we have explicitly separated out this singular part from the rest of the integral. Had we chosen to pick up the $k_{2}^{-}$pole instead of the $k_{1}^{-}$pole, there would be an overall factor of $(1-x)$ in the denominator and the endpoint singularity would occur at $x=1$. However, thus far we have only accounted for the residue part of the integration. We have yet to include the contribution along the arc that is used to close the integration contour as $k^{-} \rightarrow \infty$. To see that this contribution will not simply be zero, examine Eq. (4) and note that for the minus component the integrand behaves like $1 / k^{-}$for $k^{-} \rightarrow \infty$. Thus, the $k^{-}$integral is logarithmically divergent. As $k^{-} \rightarrow \infty$, the integrand does not fall off quickly enough to ensure that the arc contribution will vanish. Consequently, there will be non- 
zero arc contributions in all three regions of the $k^{+}$integration. This situation did not occur for the plus component since, in that case, there were sufficient powers of $k^{-}$in the denominator to ensure that the contribution along the arc at infinity vanished.

In order to recover the covariant result for the minus component of the vector two-point function, it seems that one simply has to include the contributions along the arc at infinity. Unfortunately, it turns out that explicitly evaluating the arc contributions is, in general, not a simple matter. The way in which one would usually proceed would be to substitute $k^{-}=R e^{i \phi}$ for the minus component occurring in Eq. (4),

$$
\begin{aligned}
V_{\mathrm{arc}}^{-}= & \lim _{R \rightarrow \infty}\left[\frac{1}{2} \int \frac{d k^{+}}{k^{+}\left(k^{+}-p^{+}\right)} \int d \phi\left(i R e^{i \phi}\right)\right. \\
& \left.\times \frac{R e^{i \phi}}{\left(R e^{i \phi}-k_{1}^{-}\right)\left(R e^{i \phi}-k_{2}^{-}\right)}\right],
\end{aligned}
$$

where $\phi$ runs from $0 \rightarrow \pi$ if the contour is closed in the upper half-plane and from $0 \rightarrow-\pi$ if it is closed in the lower half-plane. The $\phi$ integration is not easy to evaluate. The only straightforward way to compute this would be to neglect $k_{1}^{-}$and $k_{2}^{-}$as $R \rightarrow \infty$. The $\phi$ integration then becomes trivial. However, there are specific points $\left(k^{+}=\right.$ 0 and $k^{+}=p^{+}$) at which $k_{1}^{-}$and $k_{2}^{-}$themselves tend to infinity, and it is therefore not certain that neglecting them is safe. To see that this is indeed the case, we proceed in this straightforward way. The arc contributions for each of the three regions of $k^{+}$are then found to be

$$
\begin{gathered}
k^{+}<0: I_{\mathrm{LF}^{-}}^{\mathrm{arc}}=\frac{i \pi}{2 p^{2}} \int_{-\infty}^{0} \frac{d x}{x(1-x)}, \\
0 \leq k^{+} \leq p^{+}: I_{\mathrm{LF}^{-}}^{\operatorname{arc} 2}=\frac{i \pi}{2 p^{2}} \int_{0}^{1} \frac{d x}{x(1-x)}, \\
k^{+}>p^{+}: I_{\mathrm{LF}^{-}}^{\operatorname{arc} 3}=\frac{-i \pi}{2 p^{2}} \int_{1}^{\infty} \frac{d x}{x(1-x)} .
\end{gathered}
$$

Since $\int_{-\infty}^{\infty}+\int_{\text {arc }}=\Sigma$ (Residues), these arc contributions must be subtracted from the sum of the residues to give the final result. Conveniently, if we let $x \rightarrow(1-x)$ in Eq. (9) and add it to Eq. (11), these two terms cancel out exactly. So the only remaining contribution is that of Eq. (10). This remaining contribution can be rewritten in the following way:

$$
\begin{aligned}
I_{\mathrm{LF}^{-}}^{\mathrm{arc} 2} & =\frac{i \pi}{2 p^{2}} \int_{0}^{1} \frac{d x}{x(1-x)}=\frac{i \pi}{2 p^{2}} \int_{0}^{1} d x\left(\frac{1}{x}+\frac{1}{1-x}\right) \\
& =\frac{i \pi}{2 p^{2}} \int_{0}^{1} d x\left(\frac{1}{x}+\frac{1}{x}\right)=\frac{i \pi}{p^{2}} \int_{0}^{1} \frac{d x}{x} .
\end{aligned}
$$

Note that this arc contribution is exactly equal to the singular part of Eq. (7). Subtracting the arc contribution of Eq. (12) from the residue given by Eq. (7) gives

$$
I_{\mathrm{LF}^{-}}^{\mathrm{arc} \text { method }}=-\frac{i \pi}{p^{2}} \int_{0}^{1} d x \frac{(1-x) p^{2}+m_{1}^{2}-m_{2}^{2}}{D_{\mathrm{cov}}} .
$$

The difference between this integral and the covariant result, Eq. (2), is easily found to be $\left(i \pi / p^{2}\right) \ln \left(m_{1}^{2} / m_{2}^{2}\right)$. So this straightforward method of computing the arc contributions does not restore the equivalence between the lightfront and manifestly covariant calculations. It evidently misses contributions from the two points $k^{+}=0$ and $k^{+}=$ $p^{+}$which are needed to restore this equivalence. It is interesting to note, however, that in the special case where $m_{1}=m_{2}$, Eq. (13) does reduce to the covariant result (i.e., Eq. (2) with $m_{1}=m_{2}$ ). The reason for this will become clear later. For now we will simply state that, in the most general case, the straightforward method of evaluating the arc contributions, which involves neglecting the pole terms as $k^{-} \rightarrow \infty$, does not work.

Because of these difficulties, one might be tempted to think that somehow the minus component of the vector two-point function is not well defined in the light front. However, it is worth noting that all of the problems associated with evaluating this function vanish upon switching the order of integration. If one first carries out the integration in the complex $k^{+}$plane, and then subsequently performs the $k^{-}$integration, one obtains the manifestly covariant result without any difficulty whatsoever. If the integral is well defined for one order of integration, we maintain that it must also be well defined for the opposite ordering. So the problem is not that the overall integral is somehow ambiguous. Neither is the difficulty necessarily due to the presence of arc contributions. The true difficulty with the minus component of the vector two-point function seems to lie with the behavior of the integrand near the points $k^{+}=0$ and $k^{+}=p^{+}$. Because of the presence of the $k^{-}$term in the numerator, the $k^{-}$integration is already logarithmically divergent. However, when $k^{+}=0$ or $k^{+}=$ $p^{+}$, the $k^{-}$integration becomes linearly divergent. We must somehow find a way to deal with these strong divergences and evaluate the contributions coming from these special points.

\section{B. Asymptotic method}

Rather than attempt to explicitly evaluate the arc contributions for the minus component of the vector two-point function, we will take an alternative approach which involves dealing directly with the linear divergences present as $k^{+} \rightarrow 0$ and as $k^{+} \rightarrow p^{+}$. By identifying the behavior of the integrand at asymptotic values of $k^{-}$, we isolate the divergent parts. Hence the name "asymptotic method."

To illustrate in detail how to compute $I_{\mathrm{LF}^{-}}$in this approach, we will now return to the minus component of the vector two-point function, 


$$
V_{k_{1}(k-p)_{2}}^{-}=\frac{1}{2} \int d k^{+} d k^{-} \frac{k^{-}}{D_{1} D_{2}} .
$$

Note that as $k^{-} \rightarrow \infty$ there are linear divergences as $k^{+} \rightarrow$ 0 and as $k^{+} \rightarrow p^{+}$. Let us isolate the singular pieces in each of these two cases. First, as $k^{-} \rightarrow \infty$ and $k^{+} \rightarrow 0$,

$$
\begin{aligned}
V_{k_{1}(k-p)_{2}}^{-} & \rightarrow \frac{1}{2} \int d k^{+} d k^{-} \frac{k^{-}}{D_{1}\left(-p^{+} k^{-}\right)} \\
& =-\frac{1}{2 p^{2}} \int d k^{+} d k^{-} \frac{p^{-}}{D_{1}} \equiv V_{\text {asy } 1}^{-} .
\end{aligned}
$$

Second, in the case where $k^{-} \rightarrow \infty$ and $k^{+} \rightarrow p^{+}$,

$$
\begin{aligned}
V_{k_{1}(k-p)_{2}}^{-} & \rightarrow \frac{1}{2} \int d k^{+} d k^{-} \frac{k^{-}}{\left(p^{+} k^{-}\right) D_{2}} \\
& =\frac{1}{2 p^{2}} \int d k^{+} d k^{-} \frac{p^{-}}{D_{2}} \equiv V_{\text {asy } 2 .}^{-} .
\end{aligned}
$$

Then subtracting the two singular pieces from the vector two-point function and subsequently adding them back gives,

$$
\begin{aligned}
V_{k_{1}(k-p)_{2}=}^{-} & \frac{1}{2} \int d k^{+} d k^{-} \frac{k^{-}}{D_{1} D_{2}}-V_{\text {asy } 1}^{-}-V_{\text {asy } 2}^{-} \\
& +V_{\text {asy } 1}^{-}+V_{\text {asy } 2}^{-} \\
= & \frac{p^{-}}{2 p^{2}} \int d k^{+} d k^{-}\left[\frac{p^{+} k^{-}}{D_{1} D_{2}}+\frac{1}{D_{1}}-\frac{1}{D_{2}}\right] \\
& +V_{\text {asy } 1}^{-}+V_{\text {asy 2 }}^{-} \\
= & \frac{p^{-}}{2 p^{2}} \int d k^{+} d k^{-}\left[\frac{p^{+} k^{-}+D_{2}-D_{1}}{D_{1} D_{2}}\right] \\
& +V_{\text {asy } 1}^{-}+V_{\text {asy 2 }}^{-} \\
= & \frac{p^{-}}{2 p^{2}} \int d k^{+} d k^{-}\left[\frac{-k^{+} p^{-}+p^{2}+m_{1}^{2}-m_{2}^{2}}{D_{1} D_{2}}\right] \\
& +V_{\text {asy } 1}^{-}+V_{\text {asy } 2 .}^{-}
\end{aligned}
$$

Notice that the term in square brackets is now regular. The $k^{-}$that was in the numerator has been canceled and replaced with a $k^{+}$. As a result, the integrand now falls off sufficiently fast as $k^{-} \rightarrow \infty$ so is free from any contributions from the arc at infinity. Performing the pole integration for this term is now straightforward and gives,

$$
\begin{aligned}
V_{k_{1}(k-p)_{2}}^{-}= & \frac{-i \pi p^{-}}{p^{2}} \int_{0}^{1} d x \frac{(1-x) p^{2}+m_{1}^{2}-m_{2}^{2}}{D_{\text {cov }}} \\
& +V_{\text {asy } 1}^{-}+V_{\text {asy } 2 .}^{-}
\end{aligned}
$$

Now, the problem that remains is to evaluate the two singular pieces, $V_{\text {asy } 1}^{-}$and $V_{\text {asy } 2}^{-}$. Some of the same difficulties that were present with the vector two-point function are also present with these scalar one-point functions, or tadpoles. First, these tadpoles contain arc contributions of their own. Also, as $k^{+} \rightarrow 0$ in $V_{\text {asy } 1}^{-}$, or as $k^{+} \rightarrow p^{+}$in $V_{\text {asy } 2}^{-}$the integrals become linearly divergent. Therefore, rather than attempting to evaluate these tadpole contributions with the standard pole integration, we rewrite them in light-front cylindrical coordinates: $k^{+}=R \cos \phi$ and $k^{-}=$ $R \sin \phi$. Performing this type of variable change is perfectly safe since, although the integrals are individually divergent, their sum is finite. Evaluating these integrals over a finite region first $(R=$ finite), and then taking the limit $R \rightarrow \infty$ yields results consistent with the results obtained in a manifestly covariant calculation. For the full details of these calculations, see the appendix. The final results are summarized here:

$$
\begin{gathered}
V_{\text {asy } 1}^{-} \sim \frac{p^{-}}{p^{2}}\left[i \pi \ln \left(R^{2}\right)+\frac{\pi^{2}}{2}-i \pi \ln \left(m_{1}^{2}\right)-\mathcal{O}\left(\frac{1}{R^{4}}\right)\right], \\
V_{\text {asy } 2}^{-} \sim \frac{p^{-}}{p^{2}}\left[-i \pi \ln \left(R^{2}\right)-\frac{\pi^{2}}{2}+i \pi \ln \left(m_{2}^{2}\right)+\mathcal{O}\left(\frac{1}{R^{4}}\right)\right] .
\end{gathered}
$$

Adding $V_{\text {asy } 1}^{-}$and $V_{\text {asy } 2}^{-}$, the constants and $R$-dependent terms cancel giving

$$
V_{\text {asy } 1}^{-}+V_{\text {asy } 2}^{-}=-\frac{i \pi p^{-}}{p^{2}} \ln \left(\frac{m_{1}^{2}}{m_{2}^{2}}\right) .
$$

So substituting Eq. (20) into Eq. (18) and dividing by $p^{-}$ we obtain

$$
\begin{aligned}
I_{\mathrm{LF}^{-}}^{\text {asyethod }}= & -\frac{i \pi}{p^{2}} \int_{0}^{1} d x \frac{(1-x) p^{2}+m_{1}^{2}-m_{2}^{2}}{D_{\mathrm{cov}}} \\
& +\frac{i \pi}{p^{2}} \ln \left(\frac{m_{1}^{2}}{m_{2}^{2}}\right) .
\end{aligned}
$$

Note that the first term of this result is identical to Eq. (13) which was found using the straightforward method of evaluating the arc contributions. As already mentioned below Eq. (13), the term with the explicit logarithm makes this result identical to the manifestly covariant result of Eq. (2). The explicit logarithm term in the above result gives the contributions coming from the points $k^{+}=0$ and $k^{+}=p^{+}$. We will call these the "point contributions" to the vector two-point function. Therefore, this alternative approach has accounted for all of the contributions that were neglected by the naive pole integration technique, and which are needed in order to demonstrate the equivalence between the light-front and manifestly covariant results.

It is amusing to see that a logarithmic correction occurs, similar to Burkardt's kinetic mass counterterm [6]. However, as we use the masses occurring in the Lagrangian and do not introduce kinetic and vertex masses, we have no use for a counterterm in our perturbative calculation. Still, the corrections we find restore covariance in the same way as the kinetic mass counterterm does in Burkardt's investigation.

Recall that in Sec. II A, we showed that in the special case where the propagator masses are equal, the straight- 
forward method of computing the arc contributions was sufficient to restore the equivalence between the light-front and manifestly covariant formalisms. We are now in a position to see why this is so. Examining the case where the propagator masses are equal $\left(m_{1}=m_{2} \equiv m\right)$, the natural log term in Eq. (21) vanishes, causing Eq. (21) to reduce to Eq. (13), the result for $I_{\mathrm{LF}^{-}}$found by explicitly computing the arc contributions in the straightforward manner. In this special case, the point contributions from $k^{+}=0$ and $k^{+}=p^{+}$cancel out exactly. Therefore, the arc contributions computed in the straightforward manner are all that are needed to restore the equivalence between the lightfront and manifestly covariant formalisms. At this stage, it is not clear that such cancellations will always occur, and the fact that they do in this case should be viewed as a coincidence. The safest way to handle similar light-front integrals is to explicitly treat the point contributions as we do here.

\section{Tadpole method}

We may avoid the arc contributions altogether by using some well-known results from distribution theory [8]. The specific method we choose was pioneered by Ligterink [4]. This method reproduces a well-known result by Yan [9]. Moreover, it also reproduces the logarithmic part of the tadpole which is given by Ref. [7].

First we explain Ligterink's method. Consider the onepoint function

$$
A_{0}=\int d^{2} k \frac{1}{k^{2}-m^{2}+i \epsilon} .
$$

This object would vanish identically if one would apply the residue theorem in a naive way to the LF calculation, although dimensional regularization gives a definite result [7]. We perform the following manipulations

$$
\begin{aligned}
A_{0}= & \frac{1}{2} \int_{-\infty}^{\infty} d k^{+} \int_{-\infty}^{\infty} d k^{-} \frac{1}{k^{+} k^{-}-m^{2}+i \epsilon} \\
= & \frac{1}{2} \int_{\infty}^{\infty} d k^{+} \int_{-\infty}^{\infty} \frac{d u}{u} \frac{1}{k^{+}-\left(m^{2}-i \epsilon\right) u} \\
= & \frac{1}{2} \int_{-\infty}^{\infty} d k^{+} \int_{-\infty}^{\infty} d u \frac{1}{2}\left(\frac{1}{u+i \delta}+\frac{1}{u-i \delta}\right) \\
& \times \frac{1}{k^{+}-\left(m^{2}-i \epsilon\right) u},
\end{aligned}
$$

where in the second line we performed the transformation $u=1 / k^{-}$and in the last line split the $1 / u$ denominator into two poles off the real $u$ axis. This split produces just the correct result, as we shall see. Before applying this form to our integrals, we make a final step for the tadpole. After the $k^{-}$integration has been carried out, which can be done safely using the residue theorem as the integral over $u$ is convergent, a divergent $k^{+}$integral appears that must be regulated. We do that by using a finite cutoff. The result is

$$
\begin{aligned}
A_{0} & =\frac{1}{2} \int_{-\infty}^{\infty} d k^{+}\left[-i \pi \frac{\theta\left(k^{+}\right)}{k^{+}+i \delta m^{2}}+i \pi \frac{\theta\left(-k^{+}\right)}{k^{+}-i \delta m^{2}}\right] \\
& =\lim _{\Lambda \rightarrow \infty} \int_{0}^{\Lambda} d k^{+} \frac{-i \pi}{k^{+}+i \delta m^{2}}=-i \pi\left(\ln (\Lambda / i \delta)-\ln m^{2}\right) .
\end{aligned}
$$

This may be compared to the result from dimensional regularization [7]

$$
A_{0}=-i \pi\left(\frac{1}{\epsilon}-\gamma+\ln 4 \pi-\ln \left(m^{2} / \mu^{2}\right)\right)+\mathcal{O}(\epsilon) .
$$

Clearly, the correct dependence on the mass of the covariant amplitude is reproduced by the LF calculation.

In order to demonstrate that this method works for $V^{+}$ too, we redo the calculation as follows:

$$
\begin{aligned}
V^{+}= & \frac{1}{2} \int_{-\infty}^{\infty} d k^{+} \frac{k^{+}}{k^{+}\left(k^{+}-p^{+}\right)} \frac{1}{k_{1}^{-}-k_{2}^{-}} \\
& \times \int_{-\infty}^{\infty} d k^{-}\left[\frac{1}{k^{-}-k_{1}^{-}}-\frac{1}{k^{-}-k_{2}^{-}}\right] \\
= & \frac{1}{2} \int_{-\infty}^{\infty} d k^{+} \frac{1}{k_{1}^{-}-k_{2}^{-}}\left[\frac{k^{+}}{k^{+}-p^{+}}\right. \\
& \times \int_{-\infty}^{\infty} d k^{-} \frac{1}{k^{+} k^{-}-m_{1}^{2}+i \epsilon} \\
& \left.-\int_{-\infty}^{\infty} d k^{-} \frac{1}{\left(k^{+}-p^{+}\right)\left(k^{-}-p^{-}\right)-m_{2}^{2}+i \epsilon}\right] .
\end{aligned}
$$

Now we insert the result of the $u$ integral Eq. (24). As there occurs a factor $k^{+}$in front of the first $k^{-}$integral, the latter reduces to a finite result:

$$
\begin{aligned}
V_{\operatorname{tad} 1}^{+}= & \frac{1}{2} \int_{-\infty}^{\infty} d k^{+} \frac{k^{+}}{k^{+}\left(k^{+}-p^{+}\right)} \frac{1}{k_{1}^{-}-k_{2}^{-}}(-i \pi) \\
& \times\left[\frac{\theta\left(k^{+}\right)}{k^{+}+i \delta m_{1}^{2}}-\frac{\theta\left(-k^{+}\right)}{k^{+}-i \delta m_{1}^{2}}\right]
\end{aligned}
$$

As $k_{2}^{-} \rightarrow \infty$ for $k^{+} \rightarrow p^{+}$, this integral is nonsingular. The final result is

$$
V_{\operatorname{tad} 1}^{+}=-\frac{i \pi}{2}\left\{\int_{-\infty}^{0} d k^{+} \frac{-k^{+}}{D_{\mathrm{LF}}}+\int_{0}^{\infty} d k^{+} \frac{k^{+}}{D_{\mathrm{LF}}}\right\} .
$$

The function $D_{\mathrm{LF}}$ is defined by

$$
\begin{aligned}
D_{\mathrm{LF}} & =k^{+}\left(p^{+}-k^{+}\right) p^{-}-\left(p^{+}-k^{+}\right) m_{1}^{2}-k^{+} m_{2}^{2} \\
& =p^{+} D_{\mathrm{cov}}
\end{aligned}
$$

In a similar way we calculate the second part 


$$
\begin{aligned}
V_{\operatorname{tad} 2}^{+}= & \frac{1}{2} \int_{-\infty}^{\infty} d k^{+} \frac{k^{+}\left(k^{+}-p^{+}\right)}{D_{\mathrm{LF}}}(-i \pi) \\
& \times\left[\frac{\theta\left(k^{+}-p^{+}\right)}{k^{+}-p^{+}+i \delta m_{2}^{2}}-\frac{\theta\left(p^{+}-k^{+}\right)}{k^{+}-p^{+}-i \delta m_{2}^{2}}\right] \\
= & -\frac{i \pi}{2}\left\{\int_{-\infty}^{p^{+}} d k^{+} \frac{-k^{+}}{D_{\mathrm{LF}}}+\int_{p^{+}}^{\infty} d k^{+} \frac{k^{+}}{D_{\mathrm{LF}}}\right\} .
\end{aligned}
$$

We see that the two contributions contain integrals that cancel on the intervals $(-\infty, 0)$ and $\left(p^{+}, \infty\right)$, which is in agreement, of course, with the standard method that is based on the residue theorem. The total result is

$$
\begin{aligned}
V^{+} & =V_{\operatorname{tad} 1}^{+}-V_{\operatorname{tad} 2}^{+}=-i \pi \int_{0}^{p^{+}} d k^{+} \frac{k^{+}}{D_{\mathrm{LF}}} \\
& =-i \pi \int_{0}^{1} d x \frac{x}{D_{\mathrm{cov}}} .
\end{aligned}
$$

Because the $k^{-}$integral is nonsingular in the case of $V^{+}$, the full power of the splitting Eq. (23) is not needed. As we now turn to $V^{-}$, we shall see that this method produces the covariant result and also kills the integrals over the intervals $(-\infty, 0)$ and $\left(p^{+}, \infty\right)$ :

$$
\begin{aligned}
V^{-}= & \frac{1}{2} \int_{-\infty}^{\infty} d k^{+} \frac{1}{k^{+}\left(k^{+}-p^{+}\right)} \\
& \times \int_{-\infty}^{\infty} d k^{-} \frac{k^{-}}{\left(k^{-}-k_{1}^{-}\right)\left(k^{-}-k_{2}^{-}\right)} \\
= & \frac{1}{2} \int_{-\infty}^{\infty} d k^{+} \frac{1}{D_{\mathrm{LF}}} \int_{-\infty}^{\infty} d k^{-}\left[\frac{k_{1}^{-}}{k^{-}-k_{1}^{-}}-\frac{k_{2}^{-}}{k^{-}-k_{2}^{-}}\right] .
\end{aligned}
$$

Using the same procedure as before we can evaluate the $k^{-}$integrals to find

$$
\begin{aligned}
V_{\operatorname{tad} 1}^{-}= & \frac{1}{2} \int_{-\infty}^{\infty} d k^{+} \frac{m_{1}^{2}}{D_{\mathrm{LF}}}(-i \pi)\left[\frac{\theta\left(k^{+}\right)}{k^{+}+i \delta m_{1}^{2}}\right. \\
& \left.-\frac{\theta\left(-k^{+}\right)}{k^{+}-i \delta m_{1}^{2}}\right], \\
V_{\operatorname{tad} 2}^{-}= & \frac{1}{2} \int_{-\infty}^{\infty} d k^{+} \frac{\left(k^{+}-p^{+}\right) p^{-}+m_{2}^{2}}{D_{\mathrm{LF}}}(-i \pi) \\
& \times\left[\frac{\theta\left(k^{+}-p^{+}\right)}{k^{+}-p^{+}+i \delta m_{2}^{2}}-\frac{\theta\left(p^{+}-k^{+}\right)}{k^{+}-p^{+}-i \delta m_{2}^{2}}\right] .
\end{aligned}
$$

The part containing the factor $\left(k^{+}-p^{+}\right) p^{-}$is regular and can be evaluated immediately

$$
V_{\operatorname{tad} 2 p^{-}}^{-}=-\frac{i \pi}{2} p^{-}\left[\int_{-\infty}^{p^{+}} d k^{+} \frac{-1}{D_{\mathrm{LF}}}+\int_{p^{+}}^{\infty} d k^{+} \frac{1}{D_{\mathrm{LF}}}\right] .
$$

The other contributions can be rewritten such that the singular integrals are separated. We do so by writing

$$
\begin{aligned}
\frac{1}{D_{\mathrm{LF}}} & =\left[\frac{1}{D_{\mathrm{LF}}}+\frac{1}{m_{1}^{2} p^{+}}\right]-\frac{1}{m_{1}^{2} p^{+}} \\
& =k^{+} \frac{\left(p^{+}-k^{+}\right) p^{-}+m_{1}^{2}-m_{2}^{2}}{m_{1}^{2} p^{+} D_{\mathrm{LF}}}-\frac{1}{m_{1}^{2} p^{+}} \\
& =\left[\frac{1}{D_{\mathrm{LF}}}+\frac{1}{m_{2}^{2} p^{+}}\right]-\frac{1}{m_{2}^{2} p^{+}} \\
& =\left(p^{+}-k^{+}\right) \frac{k^{+} p^{-}-m_{1}^{2}+m_{2}^{2}}{m_{2}^{2} p^{+} D_{\mathrm{LF}}}-\frac{1}{m_{2}^{2} p^{+}} .
\end{aligned}
$$

The remaining integrals are now split accordingly. Using an obvious notation we write

$$
\begin{aligned}
V_{\mathrm{tad} 1 \mathrm{R}}^{-}= & -\frac{i \pi}{2} m_{1}^{2} \int_{-\infty}^{\infty} d k^{+} \frac{\left(p^{+}-k^{+}\right) p^{-}+m_{1}^{2}-m_{2}^{2}}{m_{1}^{2} p^{+} D_{\mathrm{LF}}} \\
& \times\left[\frac{k^{+} \theta\left(k^{+}\right)}{k^{+}+i \delta m_{1}^{2}}-\frac{k^{+} \theta\left(-k^{+}\right)}{k^{+}-i \delta m_{1}^{2}}\right] \\
= & -\frac{i \pi}{2} \frac{1}{p^{+}}\left[-\int_{-\infty}^{0} d k^{+} \frac{\left(p^{+}-k^{+}\right) p^{-}+m_{1}^{2}-m_{2}^{2}}{D_{\mathrm{LF}}}\right. \\
& \left.+\int_{0}^{\infty} d k^{+} \frac{\left(p^{+}-k^{+}\right) p^{-}+m_{1}^{2}-m_{2}^{2}}{D_{\mathrm{LF}}}\right]
\end{aligned}
$$

The other piece becomes

$$
\begin{aligned}
V_{\operatorname{tad} 2 \mathrm{R}}^{-}= & -\frac{i \pi}{2} \frac{1}{p^{+}}\left[-\int_{-\infty}^{p^{+}} d k^{+} \frac{-k^{+} p^{-}+m_{1}^{2}-m_{2}^{2}}{D_{\mathrm{LF}}}\right. \\
& \left.+\int_{p^{+}}^{\infty} d k^{+} \frac{-k^{+} p^{-}+m_{1}^{2}-m_{2}^{2}}{D_{\mathrm{LF}}}\right] .
\end{aligned}
$$

The singular integrals are

$$
\begin{aligned}
V_{\operatorname{tad} 1 \mathrm{~S}}^{-}= & \frac{i \pi}{2} \frac{1}{p^{+}} \int_{-\infty}^{\infty} d k^{+}\left[\frac{\theta\left(k^{+}\right)}{k^{+}+i \delta m_{1}^{2}}-\frac{\theta\left(-k^{+}\right)}{k^{+}-i \delta m_{1}^{2}}\right] \\
V_{\operatorname{tad} 2 \mathrm{~S}}^{-}= & \frac{i \pi}{2} \frac{1}{p^{+}} \int_{-\infty}^{\infty} d k^{+}\left[\frac{\theta\left(k^{+}-p^{+}\right)}{k^{+}-p^{+}+i \delta m_{2}^{2}}\right. \\
& \left.-\frac{\theta\left(p^{+}-k^{+}\right)}{k^{+}-p^{+}-i \delta m_{2}^{2}}\right] .
\end{aligned}
$$

Now we combine all the contributions. We have $V^{-}=$ $V_{\operatorname{tad} 1 \mathrm{R}}^{-}+V_{\operatorname{tad} 1 \mathrm{~S}}^{-}-V_{\operatorname{tad} 2 p^{-}}^{-}-V_{\operatorname{tad} 2 \mathrm{R}}^{-}-V_{\operatorname{tad} 2 \mathrm{~S}}^{-}$. We add the regular pieces in the different domains (i) $k^{+}<0$, (ii) $0<$ $k^{+}<p^{+}$, and (iii) $k^{+}>p^{+}$. Then we find 


$$
\begin{aligned}
& V_{\text {tad (i)R }}^{-}=-\frac{i \pi}{2 p^{+}} \int_{-\infty}^{0} d k^{+} \frac{-p^{+} p^{-}+\left(p^{+}-k^{+}\right) p^{-}+m_{1}^{2}-m_{2}^{2}+k^{+} p^{-}-m_{1}^{2}+m_{2}^{2}}{D_{\mathrm{LF}}}=0, \\
& V_{\operatorname{tad}(\mathrm{iii}) \mathrm{R}}^{-}=-\frac{i \pi}{2 p^{+}} \int_{p^{+}}^{\infty} d k^{+} \frac{p^{+} p^{-}-\left(p^{+}-k^{+}\right) p^{-}-m_{1}^{2}+m_{2}^{2}-k^{+} p^{-}+m_{1}^{2}-m_{2}^{2}}{D_{\mathrm{LF}}}=0 .
\end{aligned}
$$

Clearly, we are left with the regular contribution from domain (ii) plus the singular contributions. The former is given by

$$
V_{\operatorname{tad} \mathrm{R}}^{-}=-\frac{i \pi}{p^{+}} \int_{0}^{p^{+}} d k^{+} \frac{\left(p^{+}-k^{+}\right) p^{-}+m_{1}^{2}-m_{2}^{2}}{D_{\mathrm{LF}}} .
$$

The latter are evaluated using the technique that lead to Eq. (24) and give

$$
\begin{aligned}
V_{\operatorname{tad} 1 \mathrm{~S}}^{-} & =\frac{i \pi}{p^{+}} \int_{0}^{\infty} d k^{+} \frac{1}{k^{+}+i \delta m_{1}^{2}} \\
& =\frac{i \pi}{p^{+}}\left[\ln (\Lambda / i \delta)-\ln m_{1}^{2}\right], \\
V_{\operatorname{tad} 2 \mathrm{~S}}^{-} & =\frac{i \pi}{p^{+}} \int_{p^{+}}^{\infty} d k^{+} \frac{1}{k^{+}-p^{+}+i \delta m_{2}^{2}} \\
& =\frac{i \pi}{p^{+}}\left[\ln (\Lambda / i \delta)-\ln m_{2}^{2}\right] .
\end{aligned}
$$

The singular integrals add up to

$$
V_{\text {tad S }}^{-}=\frac{i \pi}{p^{+}} \ln \left(m_{2}^{2} / m_{1}^{2}\right) \text {. }
$$

Now we can check that the sum of the regular and the singular parts is equal to the covariant result. To do so, we consider the integral

$$
\begin{aligned}
I & =\frac{i \pi}{p^{+}} \int_{0}^{p^{+}} d k^{+} \frac{\left(p^{+}-2 k^{+}\right) p^{-}+m_{1}^{2}-m_{2}^{2}}{D_{\mathrm{LF}}} \\
& =\frac{i \pi}{p^{+}} \int_{0}^{p^{+}} d k^{+} \frac{d D_{\mathrm{LF}} / d k^{+}}{D_{\mathrm{LF}}}=\frac{i \pi}{p^{+}} \ln \left(m_{2}^{2} / m_{1}^{2}\right) .
\end{aligned}
$$

Thus, we add $I$ to $V_{\mathrm{R}}^{-}$to find

$$
V^{-}=-\frac{i \pi}{p^{+}} \int_{0}^{p^{+}} d k^{+} \frac{k^{+} p^{-}}{D_{\mathrm{LF}}}=-i \pi p^{-} \int_{0}^{1} d x \frac{x}{D_{\mathrm{cov}}},
$$

which is indeed the covariant result.

Comparing the tadpole method with the asymptotic one, we see that the latter succeeded in calculating both the arc and the point contributions in a simpler way, because the parts $V_{\text {asy } 1,2}$ combine these contributions in a more efficient way than $V_{\operatorname{tad} 1,2}$.

\section{Similarities with the tensor method}

We now wish to show that the asymptotic method introduced in Sec. II B is very similar to the tensor method which reduces a given loop integral to a sum of scalar $n$-point functions. The tensor method is applied to the vector two-point function in the following way:

$$
\int d^{2} k \frac{k^{\mu}}{\left[k^{2}-m_{1}^{2}+i \epsilon\right]\left[(k-p)^{2}-m_{2}^{2}+i \epsilon\right]}=I p^{\mu} .
$$

Contracting both sides with $p_{\mu}$ and solving for $I$ yields

$$
I=\frac{1}{p^{2}} \int d^{2} k \frac{k \cdot p}{\left[k^{2}-m_{1}^{2}+i \epsilon\right]\left[(k-p)^{2}-m_{2}^{2}+i \epsilon\right]},
$$

where $p^{2} \neq 0$. Using the denominators $D_{1}$ and $D_{2}$, we note that $(k \cdot p)=\left(D_{1}-D_{2}+p^{2}+m_{1}^{2}-m_{2}^{2}\right) / 2$. Substituting this into Eq. (46) yields

$$
I=\frac{1}{2 p^{2}} \int d^{2} k\left[\frac{1}{D_{2}}-\frac{1}{D_{1}}+\frac{p^{2}+m_{1}^{2}-m_{2}^{2}}{D_{1} D_{2}}\right] .
$$

The expression for $I$ has now been reduced from a vector two-point function to a sum of scalar one-point and twopoint functions. It can be evaluated either in a manifestly covariant way or in the light front. The manifestly covariant result is given by

$$
I_{\mathrm{cov}}=-\frac{i \pi}{2 p^{2}}\left[\int_{0}^{1} d x \frac{p^{2}+m_{1}^{2}-m_{2}^{2}}{D_{\mathrm{cov}}}+\ln \left(\frac{m_{1}^{2}}{m_{2}^{2}}\right)\right],
$$

which is equivalent to Eq. (2).

To evaluate $I$ in the light front, we begin by comparing Eqs. (17) and (47). In Eq. (17) we artificially separated out the singular parts of the integrand. But note how in Eq. (47) the tensor method naturally separates out the singular terms. The two singular pieces in Eq. (17), $V_{1}^{-}$and $V_{2}^{-}$, correspond to the two scalar one-point functions in Eq. (47) in the following way:

$$
\int d^{2} k \frac{1}{D_{1}}=-\frac{p^{2}}{p^{-}} V_{1}^{-}, \quad \int d^{2} k \frac{1}{D_{2}}=\frac{p^{2}}{p^{-}} V_{2}^{-} .
$$

Taking the difference between these two scalar one-point functions gives

$$
\int d^{2} k\left[\frac{1}{D_{2}}-\frac{1}{D_{1}}\right]=\frac{p^{2}}{p^{-}}\left(V_{1}^{-}+V_{2}^{-}\right)=-i \pi \ln \left(\frac{m_{1}^{2}}{m_{2}^{2}}\right) .
$$

As for the scalar two-point function in Eq. (47), since there are no possible arc contributions the pole integration is straightforward and gives 


$$
\begin{aligned}
\int d^{2} k \frac{1}{D_{1} D_{2}} & =\frac{1}{2} \int \frac{d k^{+} d k^{-}}{k^{+}\left(k^{+}-p^{+}\right)\left[k^{-}-k_{1}^{-}\right]\left[k^{-}-k_{2}^{-}\right]} \\
& =-i \pi \int_{0}^{1} d x \frac{1}{D_{\text {cov }}} .
\end{aligned}
$$

Substituting the light-front results (50) and (51) into Eq. (47) gives

$$
I_{\mathrm{LF}}=-\frac{i \pi}{2 p^{2}}\left[\int_{0}^{1} d x \frac{p^{2}+m_{1}^{2}-m_{2}^{2}}{D_{\mathrm{cov}}}+\ln \left(\frac{m_{1}^{2}}{m_{2}^{2}}\right)\right]
$$

which is identical to the manifestly covariant result in Eq. (48).

The tensor method provides a natural way in which to separate the singular pieces from the integral being considered. Also, it seems to guarantee that, at least in $(1+1)$ dimensions, any integral that is finite when computed in a manifestly covariant way will be free of spurious divergences when computed in the light front. This can be seen from the fact that the tensor method reduces an integral to a sum of scalar $n$-point functions. In $(1+1)$ dimensions, power counting tells us that scalar two-point functions and higher are all free of spurious divergences. This leaves just the scalar one-point functions to be concerned with.
Even when evaluated in a manifestly covariant way, the scalar one-point functions are of course divergent. However, if the full manifestly covariant result is finite, then this means that the scalar one-point functions must come in pairs such that the divergent parts cancel each other out. Since, as we have shown in Sec. II B, the scalar one-point functions have a similar structure in the light front, it is reasonable to conclude that the divergent parts of these will always cancel as well.

Despite its advantages for the two-point functions that depend on a single external momentum only, one must keep in mind that in more complicated cases like the three-point function, its advantages may not be so obvious. In the next section we discuss the triangle diagram and use the asymptotic and tadpole methods only.

\section{PSEUDOSCALAR CHARGE FORM FACTOR}

\section{A. Preliminaries}

We now apply the lessons we have learned from investigating the vector two-point function to the calculation of the pseudoscalar charge form factor. The current corresponding to the diagram of Fig. 2 is given by

$$
\left\langle p^{\prime}\left|J^{\mu}\right| p\right\rangle=\frac{4 N}{(2 \pi)^{2}} \int d^{2} k \frac{k^{\mu}\left(-k^{2}+2 m_{1} m_{2}-m_{2}^{2}+p \cdot p^{\prime}\right)+p^{\mu}\left(k^{2}-k \cdot p^{\prime}-m_{1} m_{2}\right)+p^{\prime \mu}\left(k^{2}-k \cdot p-m_{1} m_{2}\right)}{D_{1} D_{2} D_{3}},
$$

where we introduced a third denominator besides the ones given in Eq. (5)

$$
D_{3}=\left(k-p^{\prime}\right)^{2}-m_{2}^{2}+i \epsilon=\left(k^{+}-p^{\prime+}\right)\left(k^{-}-p^{-}\right)-m_{2}^{2}+i \epsilon
$$

and $N$ is a normalization constant. If we define the form factor by $\left\langle p^{\prime}\left|J^{\mu}\right| p\right\rangle=i\left(p^{\mu}+p^{\prime \mu}\right) F\left(q^{2}\right)$, then in terms of the Feynman parameters $x$ and $y$ the manifestly covariant result is given by

$$
\begin{aligned}
F_{\text {cov }}\left(q^{2}\right)= & \frac{N}{\pi} \int_{0}^{1} d x \\
& \times \int_{0}^{1-x} d y \frac{[y+2 x(1-x-y)]\left(m_{2}^{2}-m_{1}^{2}\right)+(1-x)\left(m_{1}^{2}+m_{1} m_{2}\right)-x\left[(x+y-1)^{2} M^{2}-x y q^{2}+m_{1} m_{2}\right]}{\left[(x+y)(x+y-1) M^{2}-x y q^{2}+(1-x-y) m_{1}^{2}+(x+y) m_{2}^{2}\right]^{2}},
\end{aligned}
$$

where we have used $p^{2}=p^{2}=M^{2}$. We can now examine the plus and minus components of the current separately. Just as in the case of the vector two-point function, the plus component of the current contains no arc contributions and easily gives the same result as Eq. (55). However, the minus component involves an integrand that does not vanish sufficiently fast as $k^{-} \rightarrow \infty$ to avoid arc contributions. It can be written as

$$
\left\langle p^{\prime}\left|J^{-}\right| p\right\rangle=\frac{N}{2 \pi^{2}} \int d k^{+} d k^{-} \frac{-k^{+} k^{-} k^{-}-p^{-} p^{--} k^{+}+\left(2 m_{1} m_{2}-m_{2}^{2}\right) k^{-}+\left(k^{+} k^{-}-m_{1} m_{2}\right)\left(p^{-}+p^{--}\right)}{D_{1} D_{2} D_{3}} .
$$

If we evaluate this naively by taking simply the sum of the residues and neglecting the arc contributions, we obtain

$$
F_{\mathrm{LF}^{-}}^{\text {residues }}\left(q^{2}\right)=\frac{N}{\pi}\left(\frac{1+\alpha}{2+\alpha}\right)\left[\int_{0}^{1} d x \frac{T_{\mathrm{val}}^{-}}{D_{\mathrm{val}}}-\int_{0}^{\alpha} d x \frac{T_{\mathrm{nv}}^{-}}{D_{\mathrm{nv}}}+\frac{1}{\alpha M^{2}} \int_{0}^{\alpha} d x \frac{1}{\alpha-x}\right]
$$

with the numerators and denominators given by 


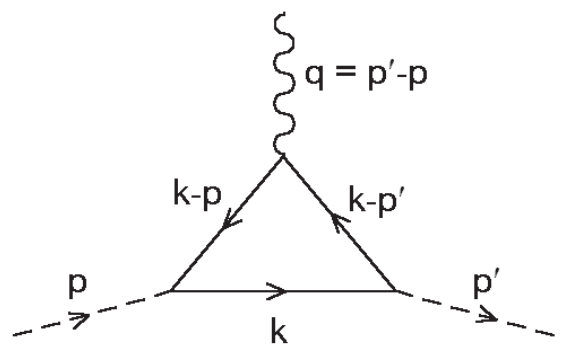

FIG. 2. The triangle diagram.

$$
\begin{aligned}
T_{\mathrm{val}}^{-}= & (1-x)^{2} M^{2}+(1+\alpha)\left(m_{1}-m_{2}\right)^{2}\left(m_{1}^{2} / M^{2}\right) \\
& +(2+\alpha)(1-x)\left(m_{1} m_{2}-m_{1}^{2}\right), \\
D_{\mathrm{val}}= & {\left[x(1-x) M^{2}-x m_{1}^{2}-(1-x) m_{2}^{2}\right] } \\
& \times\left[(1-x)(\alpha+x) M^{2}+(1-x)(1+\alpha)\left(m_{1}^{2}-m_{2}^{2}\right)\right. \\
& \left.-(1+\alpha)^{2} m_{1}^{2}\right], \\
T_{\mathrm{nv}}^{-}= & x(1+x)(\alpha-x) M^{2}-(1+\alpha)^{2}\left(m_{1}-m_{2}\right)^{2}\left(m_{2}^{2} / M^{2}\right) \\
& -(1+\alpha)(\alpha-x)\left[x\left(m_{1}^{2}-m_{2}^{2}\right)+\alpha\left(m_{2}^{2}-m_{1} m_{2}\right)\right] \\
& +(1+\alpha)^{2}(1+\alpha-x) m_{2}^{2}, \\
D_{\mathrm{nv}}= & \alpha\left[(1+x)(\alpha-x) M^{2}+(1+x)(1+\alpha)\left(m_{1}^{2}-m_{2}^{2}\right)\right. \\
& \left.-(1+\alpha)^{2} m_{1}^{2}\right]\left[x(\alpha-x) M^{2}+(1+\alpha) m_{2}^{2}\right] .
\end{aligned}
$$

We see an end-point singularity occurring at $x=\alpha$ which we explicitly separated from the rest of the integral. This end-point singularity arises because we have neglected the contributions along the arcs at infinity. We will now show how the methods of Secs. II B and IIC can be used to restore the equivalence between this light-front result and the manifestly covariant result.

\section{B. The asymptotic method}

Looking at Eq. (56), power counting in $k^{-}$tells us that the first term in the numerator is the only one that will have any arc contribution. This "bad" term by itself is

$$
\left\langle J^{-}\right\rangle_{\text {bad }}=\frac{N}{2 \pi^{2}} \int d k^{+} d k^{-} \frac{-k^{+} k^{-} k^{-}}{D_{1} D_{2} D_{3}} .
$$

Note that as $k^{-} \rightarrow \infty$, there are potential linear divergences as $k^{+} \rightarrow 0, k^{+} \rightarrow p^{+}$, and $k^{+} \rightarrow p^{\prime+}$. We will isolate these one at a time, beginning with the case where $k^{+} \rightarrow 0$ while $k^{-} \rightarrow \infty$,

$$
\left\langle J^{-}\right\rangle_{\mathrm{bad}} \rightarrow \frac{N}{2 \pi^{2}} \int d k^{+} d k^{-} \frac{-k^{+} k^{-} k^{-}}{D_{1}\left[-p^{+} k^{-}\right]\left[-p^{\prime+} k^{-}\right]}=0 .
$$

So it turns out that the potentially singular term vanishes in this case. Now, for the case in which $k^{+} \rightarrow p^{+}$as $k^{-} \rightarrow \infty$,

$$
\begin{aligned}
\left\langle J^{-}\right\rangle_{\mathrm{bad}} & \rightarrow \frac{N}{2 \pi^{2}} \int d k^{+} d k^{-} \frac{-p^{+} k^{-} k^{-}}{\left[p^{+} k^{-}\right] D_{2}\left[\left(p^{+}-p^{\prime+}\right) k^{-}\right]} \\
& =\frac{N}{2 \pi^{2}} \int d k^{+} d k^{-} \frac{1}{q^{+} D_{2}} \equiv\left\langle J^{-}\right\rangle_{2} .
\end{aligned}
$$

And finally, for the case in which $k^{+} \rightarrow p^{\prime+}$ as $k^{-} \rightarrow \infty$,

$$
\begin{aligned}
\left\langle J^{-}\right\rangle_{\text {bad }} & \rightarrow \frac{N}{2 \pi^{2}} \int d k^{+} d k^{-} \frac{-p^{\prime+} k^{-} k^{-}}{\left[p^{\prime+} k^{-}\right]\left[\left(p^{\prime+}-p^{+}\right) k^{-}\right] D_{3}} \\
& =\frac{N}{2 \pi^{2}} \int d k^{+} d k^{-} \frac{-1}{q^{+} D_{3}} \equiv\left\langle J^{-}\right\rangle_{3} .
\end{aligned}
$$

Then subtracting Eqs. (61) and (62) from Eq. (59) and subsequently adding them back gives

$$
\begin{aligned}
\left\langle J^{-}\right\rangle_{\text {bad }} & =\left\langle J^{-}\right\rangle_{\text {bad }}-\left\langle J^{-}\right\rangle_{2}-\left\langle J^{-}\right\rangle_{3}+\left\langle J^{-}\right\rangle_{2}+\left\langle J^{-}\right\rangle_{3} \\
& =\frac{N}{2 \pi^{2}} \int d k^{+} d k^{-}\left[\frac{-k^{+} k^{-} k^{-}}{D_{1} D_{2} D_{3}}-\frac{1}{q^{+} D_{2}}+\frac{1}{q^{+} D_{3}}\right]+\left\langle J^{-}\right\rangle_{2}+\left\langle J^{-}\right\rangle_{3} \\
& =\frac{N}{2 \pi^{2}} \int d k^{+} d k^{-}\left[\frac{-q^{-} k^{+} k^{+} k^{-}+m_{1}^{2} k^{+} q^{-}-m_{1}^{2} k^{-} q^{+}}{q^{+} D_{1} D_{2} D_{3}}\right]+\left\langle J^{-}\right\rangle_{2}+\left\langle J^{-}\right\rangle_{3} .
\end{aligned}
$$

Note that the first term of the last line is now regular and free of any arc contributions. Also note that $\left\langle J^{-}\right\rangle_{2}$ and $\left\langle J^{-}\right\rangle_{3}$ both have the form of Eq. (19), and they differ only by an overall sign. Since these two terms have the same mass term, $\ln \left(m_{2}^{2}\right)$, they end up completely canceling each other out when added together. Therefore, substituting Eq. (63) back into Eq. (56) gives

$$
\begin{aligned}
\left\langle p^{\prime}\left|J^{-}\right| p\right\rangle= & \frac{N}{2 \pi^{2}} \int d k^{+} d k^{-} \\
& \times \frac{\left[-k^{+} k^{+}\left(q^{-} / q^{+}\right)-\left(m_{1}-m_{2}\right)^{2}+k^{+}\left(p^{-}+p^{--}\right)\right] k^{-}+\left[m_{1}^{2}\left(q^{-} / q^{+}\right)-p^{-} p^{\prime-}\right] k^{+}-m_{1} m_{2}\left(p^{-}+p^{\prime-}\right)}{D_{1} D_{2} D_{3}} .
\end{aligned}
$$


The pole integration can now be carried out without any complications, giving for the form factor

$$
F_{\mathrm{LF}^{-}}\left(q^{2}\right)=\frac{N}{\pi}\left(\frac{1+\alpha}{2+\alpha}\right)\left[\int_{0}^{1} d x \frac{T_{\mathrm{val}}^{-}}{D_{\mathrm{val}}}-\int_{0}^{\alpha} d x \frac{T_{\mathrm{nv}}^{-}}{D_{\mathrm{nv}}}\right]
$$

with the numerators and denominator functions given before. When evaluated numerically, this light-front result for the form factor is identical to the manifestly covariant result of Eq. (55).

In retrospect, we can now see that this form factor calculation could have been done by explicitly computing the arc contributions in the straightforward way described in Sec. II A. This is because the point contributions coming from $k^{+}=p^{+}$and $k^{+}=p^{\prime+}$-Eqs. (61) and (62) respectively - cancel out exactly. To see that this is in fact the case, we have explicitly computed the arc contribution to the form factor in the straightforward way and obtained

$$
F_{\mathrm{LF}^{-}}^{\mathrm{arc}}\left(q^{2}\right)=\frac{N}{\pi}\left(\frac{1+\alpha}{2+\alpha}\right)\left[\frac{1}{\alpha M^{2}} \int_{0}^{\alpha} d x \frac{1}{\alpha-x}\right] .
$$

Note that this is equivalent to the singular part in the sum of the residues given by Eq. (57). When this arc contribution is subtracted from the sum of the residues, the part that is left over is identical to the form factor determined by our asymptotic method, Eq. (65).

\section{Tadpole method}

In order to facilitate the discussion of the tadpole method as used here, we introduce some terminology. First, we define numerator and denominator functions for the terms in Eq. (56):

$$
\begin{aligned}
N^{-} & =N_{\text {Reg }}^{-}+N_{\text {Sing, }}^{-} \\
N_{\text {Reg }}^{-} & =-k^{+} p^{-} p^{-}+D_{1}\left(p^{-}+p^{-}\right)+k^{-}\left(2 m_{1} m_{2}-m_{2}^{2}\right), \\
N_{\text {Sing }}^{-} & =-k^{+}\left(k^{-}\right)^{2} .
\end{aligned}
$$

In order to isolate the logarithmic singularities which arise due to the factor $\left(k^{-}\right)^{2}$ in the numerator, we may write the singular part of the numerator $N_{\text {Sing }}^{-}$in six different ways

$$
\begin{aligned}
N_{i}^{-}= & -k^{+}\left[\left(k^{-}-k_{i}^{-}\right)^{2}+2 k_{i}^{-}\left(k^{-}-k_{i}^{-}\right)+\left(k_{i}^{-}\right)^{2}\right], \\
N_{i j}^{-}= & -k^{+}\left[\left(k^{-}-k_{i}^{-}\right)\left(k^{-}-k_{j}^{-}\right)+k_{i}^{-}\left(k^{-}-k_{j}^{-}\right)\right. \\
& \left.+k_{j}^{-}\left(k^{-}-k_{i}^{-}\right)+k_{i}^{-} k_{j}^{-}\right] .
\end{aligned}
$$

By substitution of either of these representations of $N_{\text {Sing }}^{-}$in the expression for the current,

$$
\left\langle p^{\prime}\left|J^{-}\right| p\right\rangle=\frac{4 N}{(2 \pi)^{2}} \int d^{2} k \frac{N_{\mathrm{Reg}}^{-}+N_{\text {Sing }}^{-}}{D_{1} D_{2} D_{3}},
$$

we may isolate different tadpoles. Although the final result cannot depend on which choice is made, the actual calculations are very much simplified if one chooses to take a linear combination of $N_{12}^{-}$and $N_{13}^{-}$. The reason is that the tadpoles associated with $D_{2}$ and $D_{3}$ are identical, because they involve the same mass $m_{2}$ :

$$
\int d^{2} k \frac{1}{D_{2}}=\int d^{2} k \frac{1}{D_{3}},
$$

which, incidentally, explains why the asymptotic method as we used it in the previous subsection, is much simplified. So, we make the following subtraction of the integrand defining the singular contribution to the current matrix element

$$
\frac{N_{\text {Sing }}^{-}}{D_{1} D_{2} D_{3}}-a\left(\frac{1}{D_{2}}-\frac{1}{D_{3}}\right)
$$

where $a$ is a constant to be determined in such a way that $N_{\text {Sing }}^{-}$is canceled. If we define

$$
N_{\mathrm{SR}}^{-}=N_{\text {Sing }}^{-}+a D_{1}\left(D_{2}-D_{3}\right),
$$

then we find that $N_{\mathrm{SR}}^{-}$contains only a term linear in $k^{-}$if we choose $a=1 /\left(p^{\prime+}-p^{+}\right)=1 / q^{+}$. As the correction term gives rise to singular integrals that are exactly the same and have opposite sign, they cancel out and we are left with two integrals to which we can safely apply the residue theorem to find

$$
\begin{aligned}
& \left\langle p^{\prime}\left|J_{\mathrm{Reg}}^{-}\right| p\right\rangle=\int d k^{+} \int d k^{-} \frac{N_{\mathrm{Reg}}^{-}}{D_{1} D_{2} D_{3}}, \\
& \left\langle p^{\prime}\left|J_{\mathrm{SR}}^{-}\right| p\right\rangle=\int d k^{+} \int d k^{-} \frac{N_{\mathrm{SR}}^{-}}{D_{1} D_{2} D_{3}} .
\end{aligned}
$$

Application of the residue theorem now produces two contributions, the valence one and the nonvalence one, the former corresponding to $k^{-}=k_{1}^{-}$which annihilates $D_{1}$ and the latter to $k^{-}=k_{3}^{-}$, which annihilates $D_{3}$. From Eq. (72) it follows immediately that $N_{\mathrm{SR}}^{-}$is equal to its naive value in the valence case. In the nonvalence case we find the residue

$$
\frac{k^{+}\left(k_{3}^{-}\right)^{2}+D_{1} D_{2} / q^{+}}{\left(k^{+}-p^{\prime+}\right) D_{1} D_{2}}=\text { naive residue }+\frac{1}{q^{+}\left(k^{+}-p^{\prime+}\right)} \text {. }
$$

The part $1 /\left[q^{+}\left(k^{+}-p^{\prime+}\right)\right]$ is the correction that cancels the singularity in the naive nonvalence contribution that was found in Ref. [1]. One may check explicitly that upon taking this correction into account, the covariant result is recovered.

\section{SUMMARY AND CONCLUDING REMARKS}

We have demonstrated that the spurious divergences which spoil the equivalence between the light-front and manifestly covariant formalisms are merely a result of applying the residue theorem to the LF-energy integrals in a naive way. However, we have also demonstrated that computing the arc and point contributions explicitly is not necessarily an easy task. In general, a simple straightfor- 
ward computation of these arc contributions can miss other important contributions coming from specific points in the range of integration, which we have called point contributions. Because of this, we have investigated different methods of evaluating these tricky integrals, each of which ensures that all the relevant contributions will be included. Once all these contributions are properly accounted for, the spurious divergences disappear and the equivalence between the light-front and manifestly covariant results is restored.

In summary, the final answer is obtained in two steps: First the result of the naive application of the residues theorem is corrected by the arc contributions, and second the naive arc contributions are corrected by the effects of the moving poles, i.e. the point contributions. The necessity of self-induced inertias discussed in normal ordered LF Hamiltonians [5] corresponds to our first step of finding the necessity of arc contribution. Burkardt's finding $\Delta m_{\text {kin }}^{2}$ [6] as a finite contribution consistent with the parity requirement corresponds to our second step of finding a finite point contribution. The finite point contribution we find has a logarithmic dependence on the mass, similar to Burkardt's $\Delta m_{\text {kin }}^{2}$. Thus, it is conceivable that our findings may point to some deficiency in the light-front perturbation theory (LFPT) rules. It is interesting to note that if we use LFPT rules to compute the pseudoscalar charge form factor, the same end-point singularity that we have seen arise in other calculations is manifested. While it is possible to use some type of prescription to regularize this divergence, we have shown in this work that such procedures should not be needed when the integrals are handled properly. Therefore, it is entirely possible that the same deficiencies that plague other methods of calculation are also present in LFPT. Further investigations should be conducted into the nature of LFPT to see whether or not there is a need to modify the rules so as to include the necessary arc and point contributions.

Because of the rational energy-momentum dispersion relation, the light-front dynamics (LFD) has distinguished features compared to the other forms of Hamiltonian dynamics. In particular, the vacuum fluctuations are sup- pressed and the kinematic generators are proliferated in LFD. Overall, these distinguished features can be regarded as advantageous rather than as disadvantageous in the hadron phenomenology. However, in return, the LFD implies treacherous points, an example of which we present in this work. Thus, careful investigations of treacherous points and judicious ways of handling those points should be precedent for LFD to be distinctively useful compared to other forms of Hamiltonian dynamics.

\section{ACKNOWLEDGMENTS}

This work was supported in part by a grant from the U.S. Department of Energy (No. DE-FG02-96ER 40947). C. R. J. would like to thank the Department of Physics at Seoul National University for their hospitality.

\section{APPENDIX: EVALUATING THE ONE-POINT FUNCTION USING LIGHT-FRONT CYLINDRICAL COORDINATES}

The one-point function in the light front is given by

$$
A_{0}=\frac{1}{2} \int d k^{+} d k^{-} \frac{1}{k^{+} k^{-}-m^{2}+i \epsilon} .
$$

If we change to light-front cylindrical coordinates then we will have $k^{+}=r \cos \phi$ and $k^{-}=r \sin \phi$, and $A_{0}$ becomes

$$
\begin{aligned}
A_{0} & =\frac{1}{2} \int_{0}^{\infty} \int_{0}^{2 \pi} r d r d \phi \frac{1}{r^{2} \sin \phi \cos \phi-m^{2}+i \epsilon} \\
& =\frac{1}{2} \int_{0}^{\infty} \frac{d r}{r} \int_{0}^{2 \pi} d \phi \frac{1}{\sin \phi \cos \phi-m^{2} / r^{2}+i \epsilon} .
\end{aligned}
$$

Noting that $2 \sin \phi \cos \phi=\sin (2 \phi)$ and making the variable change $\theta=2 \phi$ we obtain

$$
A_{0}=\int_{0}^{\infty} \frac{d r}{r} \int_{0}^{2 \pi} d \theta \frac{1}{\sin \theta-2 m^{2} / r^{2}+i \epsilon} .
$$

We proceed by performing the integration in the complex plane. If we let $z=e^{i \theta}$ and define $\beta \equiv 2 m^{2} / r^{2}$ the integration becomes

$$
A_{0}=\int_{0}^{\infty} \frac{d r}{r} \int_{C} d z \frac{2}{\left[z-\left(i \beta+\sqrt{1-\beta^{2}}+\epsilon\right)\right]\left[z-\left(i \beta-\sqrt{1-\beta^{2}}+\epsilon\right)\right]},
$$

where $\mathrm{C}$ represents integration around the unit circle in the counterclockwise direction.

Since $\beta$ can possess any value between 0 and $\infty$ we must consider the possible cases carefully. In the case where $0 \leq$ $\beta \leq 1$, note that (neglecting $\epsilon$ momentarily) the poles lie on the unit circle in the upper half-plane. Epsilon shifts these poles in the positive direction, such that the pole in the first quadrant now lies outside the contour of integration, while the pole in the second quadrant lies within the contour of integration. Performing the integration gives

$$
A_{0}(0 \leq \beta \leq 1)=\int_{0}^{\infty} \frac{d r}{r} \frac{-2 \pi i}{\sqrt{1-\beta^{2}}} .
$$

Now, in the case where $\beta>1$, the poles move onto the imaginary axis. They are now $i\left(\beta \pm \sqrt{\beta^{2}-1}\right)+\epsilon$. Since $\beta>1$ we can write $\beta=1+\eta$. Substituting this into the poles gives $i\left(1+\eta \pm \sqrt{\eta^{2}+2 \eta}\right)+\epsilon$. Written this way it 
is clear that the pole with the minus sign lies inside the unit circle while the pole with the plus sign lies outside the unit circle. Again performing the contour integration yields

$$
A_{0}(\beta>1)=\int_{0}^{\infty} \frac{d r}{r} \frac{-2 \pi i}{\sqrt{1-\beta^{2}}} .
$$

Therefore, in both cases we obtain the same result for $A_{0}$.

Substituting back in the definition of $\beta$, and proceeding with the $r$ integration, we get

$$
\begin{aligned}
A_{0} & =-2 \pi i \int_{0}^{\infty} d r \frac{r}{\sqrt{r^{4}-4 m^{4}}} \\
& =-2 \pi i \lim _{R \rightarrow \infty} \int_{0}^{R} d r \frac{r}{\sqrt{r^{4}-4 m^{4}}} \\
& =-i \pi \lim _{R \rightarrow \infty} \operatorname{arctanh}\left(\frac{R^{2}}{\sqrt{R^{4}-4 m^{4}}}\right) \\
& =-i \pi \lim _{R \rightarrow \infty} \operatorname{arctanh}\left(\frac{1}{\sqrt{1-\left(4 m^{4} / R^{4}\right)}}\right) .
\end{aligned}
$$

Substituting a series expansion for the hyperbolic arctangent function gives the final result

$$
A_{0} \sim-i \pi \ln \left(R^{2}\right)-\frac{\pi^{2}}{2}+i \pi \ln \left(m^{2}\right)+\mathcal{O}\left(\frac{1}{R^{4}}\right) .
$$

[1] B. L. G. Bakker and C.-R. Ji, Phys. Rev. D 62, 074014 (2000).

[2] B. L. G. Bakker, H.-M. Choi, and C.-R. Ji, Phys. Rev. D 63, 074014 (2001).

[3] A. Misra and S. Warawdekar, Phys. Rev. D 71, 125011 (2005).

[4] N. E. Ligterink and B. L. G. Bakker, Phys. Rev. D 52, 5954 (1995).
[5] H.-C. Pauli and S. J. Brodsky, Phys. Rev. D 32, 1993 (1985).

[6] M. Burkardt, Phys. Rev. D 57, 1136 (1998).

[7] D. Bardin and G. Passarino, The Standard Model in the Making (Clarendon Press, Oxford, 1999).

[8] N. Ya. Vilenkin and I.M. Gelfand, Functional Analysis (Wolters-Noordhoff, Groningen, 1972).

[9] T.-M.Yan, Phys. Rev. D 7, 1780 (1973). 